\title{
The prevalence of physical activity among MBBS students in a medical college in Kerala
}

\author{
Joy $\mathrm{V}^{\mathbf{1}^{*}}$, Vincent J. ${ }^{2}$
}

DOI: https://doi.org/10.17511/ijphr.2020.i04.01

1* Vismaya Joy, Fourth-year MBBS student, Amala Institute of Medical Sciences, Thrissur, Kerala, India.

$\mathbf{2}$ Jose Vincent, Assistant Professor, Department of Community Medicine, Amala Institute of Medical Sciences, Thrissur, Kerala, India.

Background: The healthy lifestyle of medical students could facilitate the formation of healthy physicians who is more likely to give effective preventive counseling to their patients. Therefore, there is compelling evidence that the health of doctors matters, and the doctors' own physical activity practices influence their clinical attitudes towards physical activity. Aim and objectives: To assess the prevalence of physical activity and its determinants and also to find out the barriers of physical activity among MBBS students in a medical college in Kerala. Methods: A cross-sectional study was conducted amongst 180 undergraduate medical students. Data was obtained from students using the Global Physical Activity Questionnaire (GPAQ) by WHO and Metabolic Equivalents (METs) were used to express the intensity of physical activities. Results: Of the total 180 students, $128(71.1 \%)$ were found as physically active, and $52(28.9 \%)$ as physically inactive. Among the physically active students, $98(54.44 \%)$ and $30(16.66 \%)$ showed moderate and high levels of physical activity respectively. The mean MET minutes per week attained by the study population in recreational activities is more compared to work and transport. Conclusion: Of the 180 students, average MET scores attained by males and females in the 3 domains of physical activity showed significant difference highlighting the need to promote more activities among females.

Keywords: GPAQ, MBBS students, Metabolic Equivalents, Physical activity

Corresponding Author

Vismaya Joy, Fourth-year MBBS student, , Amala Institute of Medical Sciences, Thrissur, Kerala, India. Email: haivis1534joy@gmail.com
How to Cite this Article

To Browse

Joy V, Vincent J. The prevalence of physical activity among MBBS students in a medical college in Kerala. Public Health Rev Int J Public Health Res. 2020;7(4):28-34.

Available From

https://publichealth.medresearch.in/index.php/ijphr/ article/view/136

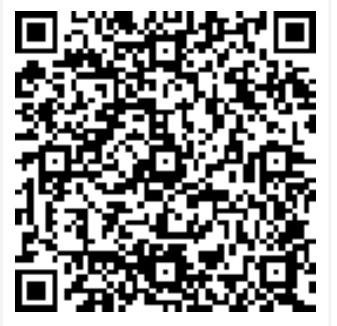

Manuscript Received 2020-08-09

Conflict of Interest No
Review Round 1 2020-08-19

Funding

$\mathrm{Nil}$

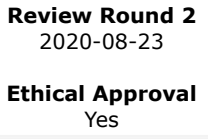

Review Round 3

Accepted 2020-08-26

Plagiarism $X$-checker $9 \%$

Note

(c) 2020 by Vismaya Joy, Jose Vincent and Published by Siddharth Health Research and Social Welfare Society. This is an Open Access article licensed under a Creative Commons Attribution 4.0 International License https://creativecommons.org/licenses/by/4.0/ unported [CC BY 4.0]. 


\section{Introduction}

The world population is aging, while increased development has been accompanied by rising disposable incomes, urbanization, mechanization, and the globalization of food markets, leading to lifestyle and behavior changes that adversely impact population health. These lifestyle changes include dietary changes (nutrition transition), reduced physical activity levels, and increased tobacco use. The result is that non-communicable diseases are increasing at alarming rates globally. The burden of non-communicable diseases in developing countries already outweighs that of communicable diseases, both in high- and low-income countries [1].

Physical activity is defined as any bodily movement produced by skeletal muscles that result in energy expenditure. The energy expenditure can be measured in kilocalories. Physical activity in daily life can be categorized into occupational, sports, conditioning, household, or other activities. Exercise is a subset of physical activity that is planned, structured, and repetitive and has as a final or an intermediate objective the improvement or maintenance of physical fitness [2].

During physical activity, whole-body oxygen consumption may increase by as much as 20 -fold, and even greater increases may occur in the working muscles. To meet its energy needs under these circumstances, skeletal muscle uses, at a greatly increased rate, its own stores of glycogen and triglycerides, as well as free fatty acids (FFAs), derived from the breakdown of adipose tissue triglycerides and glucose released from the liver. To preserve the central nervous system function, blood glucose levels are remarkably well maintained during physical activity.

Hypoglycemia during physical activity rarely occurs in non-diabetic individuals. The metabolic adjustments that preserve normoglycemia during physical activity are in large part hormonally mediated. A decrease in plasma insulin and the presence of glucagon appear to be necessary for the early increase in hepatic glucose production during physical activity, and during prolonged exercise, increases in plasma glucagon and catecholamines appear to play a key role [3].

Physical inactivity is an established risk factor for cardiovascular disease, cancer, and diabetes, which along with chronic respiratory disease account for more than $60 \%$ of all deaths [4]. Endocrinological
And metabolic effects of exercise: in well-controlled diabetic patients, physical exercise promotes the utilization of blood glucose and lowers blood glucose levels [5]. Regular physical exercise improves insulin sensitivity in lifestyle-related diseases like type 2 diabetes and hyperlipidemia $[3,5,6]$. Further, continued physical activity is associated with a higher energy turnover, with important implications for the transport, storage, and utilization of lipid fuels [7].

Chronic disease risk factors, including a sedentary lifestyle, may be present even in young children, suggesting that early prevention programs may be critical to reducing the rates of chronic disease. Accurate assessment of physical activity in children is necessary to identify current levels of activity and to assess the effectiveness of intervention programs designed to increase physical activity [8].

The healthy lifestyle of medical students could facilitate the formation of healthy physicians who is more likely to give effective preventive counseling to their patients [9]. Therefore, there is compelling evidence that the health of doctors matters and that doctors' own physical activity practices influence their clinical attitudes towards physical activity. Medical schools need to increase the proportion of students adopting and maintaining regular physical activity habits to increase the rates and quality of future physical activity counseling delivered by doctors [10].

Hence it becomes a matter of paramount importance to find out the prevalence of physical activity among MBBS students.

\section{Materials and Methods}

A cross-sectional study was conducted amongst undergraduate medical students in a medical college in Thrissur, Kerala from July 2019 to October 2019.

Sample size: The sample size was calculated from a study done by Padmapriya $\mathrm{K}$ et al in Bangalore [11]. As per the study, among 259 students, $41.3 \%$ showed high levels of physical activity. Considering the prevalence as $41.3 \%$, sample size was calculated using the formula, $n=4 p q / d 2(p=41.3 \%$, $q=100-p, d=20 \%$ of $p$ ). The minimum sample size came out to be 142 . A total of 180 students were included in the study.

Inclusion criteria: After obtaining Institutional Ethical Clearance, data was obtained from 180 students of second and third-year MBBS. 
Exclusion criteria: Physically disabled students and those who were not willing to give consent.

Consent: All the participants were briefed about the objectives of the study and informed that their participation was voluntary and was also assured of anonymity and confidentiality of the data. Written informed consent was obtained from those students willing to take part in the study.

Methodology: Data was obtained using the Global Physical Activity Questionnaire (GPAQ) by WHO and it collected information on three domains: activity at work, travel to and from places, recreational activities. All questions referred to the previous seven days. Metabolic Equivalents (METs) were used to express the intensity of physical activities. MET is the ratio of a person's working metabolic rate relative to the resting metabolic rate. One MET is defined as the energy cost of sitting quietly and is equivalent to a caloric consumption of $1 \mathrm{kcal} / \mathrm{kg} /$ hour. For the analysis of GPAQ data, existing guidelines have been adopted.

It is estimated that compared to sitting quietly, a person's caloric consumption is four times as high when being moderately active and eight times as high when being vigorously active. Weekly minutes of walking, moderate-intensity and vigorousintensity activity were calculated separately by multiplying the number of days/week by the duration on an average day. Reported minutes per week in each category were weighted by a metabolic equivalent (MET; multiples of resting energy expenditure) resulting in a physical activity estimate independent of body weight, expressed in MET-minutes/week.

Those students who have not attained WHO recommended minimal requirement of 600 MET minutes per week were classified as physically inactive, those between 600 and 3000 MET minutes per week were moderately active, and $>3000$ MET minutes per week highly active. The prevalence of physical activity was calculated as the percentage of students involved in the activity.

Height, weight, Body Mass Index (BMI) of each student along with demographic details were collected. BMI was calculated using the formulae weight divided by height in meter square and WHO Asian classification was used for analysis. Additional data was collected to find out the determinants and also the barriers of physical activity among students using a self-administered questionnaire.
Data analysis: The data collected was entered into Microsoft Excel and analysis was done by SPSS software version 20. Appropriate statistical methods were used for analysis and the results were presented as a percentage as well as mean and standard deviation (SD).

\section{Results}

Table-1: Variables and attainment of WHOrecommended MET minutes/week.

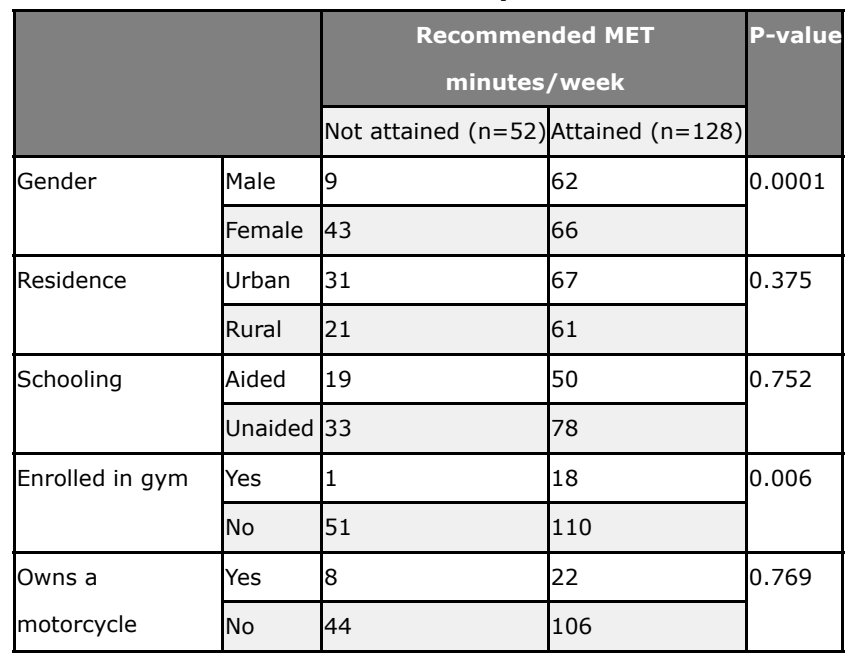

Of the total 180 study participants, 109 (60.6\%) were females, and 71 (39.4\%) males. They were within the age group 18-25. Mean height and weight were $165.32 \mathrm{~cm}$ and $59.38 \mathrm{~kg}$ respectively. The mean BMI was $21.62 \mathrm{~kg} / \mathrm{m} 2$. Out of the 180 students, 128 $(71.1 \%)$ have attained the WHO recommended MET min/week and $52(28.9 \%)$ have not. Of the total 71 males, only $9(12.68 \%)$ were physically inactive while 43 (39.44\%) out of 109 females were physically inactive and the difference was statistically significant. Of the 19 students enrolled in the gym, 18 have attained the WHO recommended MET min/week. Religion, residence, schooling, educational status of parents, whether they own a motorcycle or not does not show any statistically significant association with the level of physical activity.

Table-2: Level of physical activity attained by the study participants measured in MET minutes/week.

\begin{tabular}{|l|l|l|l|}
\hline \multirow{2}{*}{ Level of physical activity } & \multicolumn{2}{|c|}{$\begin{array}{c}\text { Frequency } \\
\text { (percentage) }\end{array}$} & \multirow{2}{*}{$\begin{array}{c}\text { Total } \\
\text { (percentage) }\end{array}$} \\
\cline { 2 - 3 } & Male & Female & \\
\hline Low (<600METmin/week) & $9(17.3)$ & $43(82.7)$ & $52(28.89)$ \\
\hline $\begin{array}{l}\text { Moderate (600- } \\
\text { 3000METmin/week) }\end{array}$ & $44(44.9)$ & $54(55.1)$ & $98(54.44)$ \\
\hline High (>3000METmin/week) & $17(56.7)$ & $13(43.3)$ & $30(16.66)$ \\
\hline
\end{tabular}


Not achieving the WHO recommended MET minutes/week of 600 was categorized as low physical activity group and $52(28.89 \%)$ fell into this category. More than half 98 (54.44\%) belonged to the group of moderate physical activity. 30 $(16.66 \%)$ study subjects belonged to those who attained high levels of physical activity. Among the 52 students who did not achieve the WHO recommended MET minutes/week, 43 (82.7\%) were females and $9(17.3 \%)$ were males. Among the 30 students who achieved high levels of MET scores, 17 $(56.7 \%)$ were males and $13(43.3 \%)$ were females.

Table-3: Mean MET minutes per week attained by study population in 3 domains of physical activity.

\begin{tabular}{|l|l|l|}
\hline \multicolumn{1}{|c|}{ Category } & \multicolumn{1}{c|}{ Mean (SD) } & \multirow{2}{*}{ P-Value } \\
\hline Work & $659.22(1297.73)$ & 0.0001 \\
\cline { 1 - 2 } Transport & $530.67(947.18)$ & \\
\hline Recreation & $1421.55(1817.54)$ & \\
\hline
\end{tabular}

When physical activity is aggregated across three domains, recreation contributes the most to the mean MET minutes per week of the study population. It was followed by work and transport contributed the least to the energy expenditure.

Different barriers to physical activity among students were assessed. Of the total participants, $128(71.1 \%)$ either agreed or strongly agreed to the statement that "Other recreational activities with my friends are more entertaining". 35 (67\%) of the 52 students who have not attained the WHO recommended MET min/week and 73 (57\%) of the 128 students who have attained the value were of the opinion that their parents gave priority to academics over physical activity. Lack of a convenient schedule was perceived as a barrier to exercise by $29(56 \%)$ students who have a low value of MET min/week. The majority of the students do not find any embarrassment in doing physical activity. They were of the opinion that exercise gave them a sense of personal accomplishment and improved the quality of their work as well as decreased feelings of stress and tension.

The present study reports the prevalence of physical activity among undergraduate medical students in a tertiary care hospital in Kerala. Of the 180 students who participated in the study, $52(28.89 \%)$ showed low levels of physical activity, 98 (54.44\%) showed moderate and $30(16.66 \%)$ showed high levels of physical activity. A similar study conducted among MBBS students in Bangalore showed that 15.4\%

\section{Discussion}

Of students did not meet the WHO recommended MET minutes/week, $43.2 \%$ of students showed moderate and $41.3 \%$ showed high levels of physical activity [11]. The percentage of students with low physical activity is much higher in the current study whereas the percentage of students with a high level of physical activity is lower compared to the study conducted in Bangalore. In the present study more than half of the students were engaged in moderate physical activity. In contrast, the study conducted in Bangalore revealed that the percentage of students engaged in moderate and high physical activity was almost the same.

The main source of physical activity energy expenditure in the present study was from recreational activities and least was from transport. In the study conducted in Bangalore, most of the students were engaged in work-related activities rather than transport and recreation. From the present study it was evident that in spite of the busy curriculum, the students were spending their time doing more recreational activities. This could be due to the presence of many physical activity programs and recreational facilities. Transport contributing least to the attainment of physical activity levels could be due to the fact that most of the students were hostellers and most of their requirements were met from their surroundings itself. Another study showed that the main contributors to total physical activity among adults were from working and active commuting. The leisure-time physical activity represented a very small proportion (9.4\%) of individuals' total activity levels [12].

One significant finding of the study is the difference between male and female students with respect to engagement in physical activity. Of the total 71 males, only $9(12.68 \%)$ were physically inactive while $43(39.44 \%)$ out of 109 females were physically inactive. This gender variation brought to light by this study is consistent with the data obtained from previous studies on physical activity among young adults. Studies quantifying physical activity and sedentary time among South Asian women showed low levels of physical activity compared with South Asian men and with white European comparison populations. The majority of studies indicated that South Asian women did not meet the recommended amounts of physical activity for health benefits. Few studies assessed sedentary 
Time. Themes emerging from qualitative studies included cultural and structural barriers to physical activity, faith and education as facilitators, and a lack of understanding of the recommended amounts of physical activity and its benefits among South Asian women [13].

In the present study, of the 19 students enrolled in the gym, 18 have attained the WHO recommended MET minutes/week and it was statistically significant ( $p$ value $=0.006$ ). A similar study conducted among health college students in Saudi Arabia revealed that no member of a sports club was a significant predictor of physical inactivity [14]. Similar results have been reported in Egypt, USA, and Hong $[15,16,17]$ because daily participation of university and community sports facilities play a crucial role in encouraging students to participate in physical activity, as the students usually do not have a full daily schedule of studies.[18] While considering the effect of parent's education on physical activity level, no significant association was found in the present study. The better awareness levels among the MBBS students about physical activity might have offset the influence of parent's educational status on attaining recommended MET scores. A similar result was obtained in the study in Saudi Arabia [14]. In contrast, a positive correlation was observed between degrees of parents' education and levels of physical activity in a study among female university students [19].

A study conducted among Egyptian and Saudi medical students showed that the most frequent barriers to physical activity were time limitation due to busy study schedules and lack of accessible and suitable sporting places [20] which did not come as a barrier in the present study. Many students in the present study considered that their parents giving priority to academics over physical activity as a barrier to doing exercise.

In the current study, more than half of the students (54.44\%) were engaged in a moderate level of physical activity. Similarly, findings from the international prevalence study on physical activity among 20 countries indicated that the majority of the population in most participating countries or regions appeared to undertake at least a moderate amount of physical activity when assessed using the multi-domain IPAQ (International Physical Activity Questionnaire). This suggests that most adults in these countries are obtaining some activity, yet the global problem of the rising prevalence of obesity remains. Thus, it appears total physical activity
In most countries remains insufficient to ensure energy balance and prevent obesity [21] or that the ratio of energy expenditure to dietary intake is unbalanced to maintain weight stability [22].

\section{Strengths and limitations of the study}

Strengths: The study topic has huge relevance in the context of non-communicable diseases. Also, the study was conducted using the WHO GPAQ questionnaire and it makes a comparison of results possible.

\section{Limitations}

The study was conducted among MBBS students in a medical college in Kerala. The results of the study may not generalizable to the general population.

\section{Conclusion}

Of the total 180 students, $52(28.89 \%)$ students did not achieve the WHO recommended MET minutes per week. 98(54.44\%) students were engaged in a moderate level of physical activity and $30(16.66 \%)$ achieved a high level of physical activity. Of the total 52 students who did not achieve the recommended MET scores, only 9 were males and the rest were females. Average MET scores attained by males and females in the 3 domains of physical activity showed significant difference highlighting the need to promote more activities among females.

Mean MET minutes per week attained by students in recreational activities were more compared to work and transport-related activities.

Those who enrolled in the health club were more physically active than others pointing to the fact that the incorporation of physical activity to the daily routine may be easy on an institutional and social platform.

Age, Body Mass Index (BMI), residence, schooling, religion, and educational status of parents were not determinants of physical activity.

\section{What does the study add to the existing knowledge}

Of the total students, $71.1 \%$ were of the opinion that other recreational activities with their friends were more entertaining than exercising. $60 \%$ of students agreed that their parents gave priority to academics over physical activity and more than half were of the opinion that exercise did not have 
Convenient schedules for them. The majority of the students felt that exercise gave them a sense of personal accomplishment and decreased feelings of stress and tension for them.

Colleges and universities should ensure a safe and conducive environment is available on college campuses for students to engage in physical activities. Health clubs and gyms should be run by the educational institution and student participation must be ensured. Promote different activities that help to integrate physical activity into routine life.

\section{Author's contribution}

Vismaya Joy: Concept, study design

Dr. Jose Vincent: Data analysis and manuscript preparation

\section{Reference}

01. Waxman A. WHO's global strategy on diet, physical activity, and health- response to a worldwide epidemic of non-communicable diseases. Scand J Nutri. 2004;48(2)58-60.

doi: $10.1080 / 11026480410033539 \quad$ [Crossref]

02. Caspersen CJ, Powell KE, Christenson GM. Physical activity, exercise, and physical fitnessdefinitions and distinctions for health-related research. Public Health Rep. 1985;100(2)126131.

[Crossref]

03. Zinman B, Ruderman N, Campaigne BN, Devlin JT, Schneider SH American Diabetes Association. Physical activity/exercise and diabetes mellitus. Diabetes Care. 2003;26(1)S73-S77.

doi: $10.2337 /$ diacare.26.2007.s73 [Crossref]

04. World Health Organization: Reducing risks, promoting healthy life. World Health Report 2002. Geneva- World Health Organization. 2002.

Available at [Article] [Crossref]

05. Trinh Oanh TH, Nguyen Nguyen D, Dibley Michael J, PhongsavanPhilayrath, Bauman Adrian E. The prevalence and correlates of physical inactivity among adults in Ho Chi Minh City. BMC Public Health. 2008; (8)204.

doi: 10.1186/1471-2458-8-204 [Crossref]
06. Sato Y. Diabetes and life-styles- Role of physical exercise for primary prevention. $\mathrm{Br} \mathrm{J}$ Nutr. 2000;84(2)S187-S190. doi: $10.1079 / 096582197388662$ [Crossref]

07. Borghouts LB, Keizer HA. Exercise and insulin sensitivity- A review. Int J Sports Med. $2000 ; 21(1) 1-12$.

doi: $10.1055 / \mathrm{s}-2000-8847$ [Crossref]

08. Haskell WL, Taylor HL, Wood PD, Schrott $H$, Heiss G. Strenuous physical activity, treadmill exercise test performance and plasma highdensity lipoprotein cholesterol. The lipid research clinics program prevalence study. Circulation.

1980;62(4 pt 2)IV53-61 [Crossref]

09. Sirard JR, Pate RR. Physical Activity Assessment in Children and Adolescents. Sports Med. $2001 ; 31(6) 439-454$.

doi: 10.2165/00007256-200131060-00004 [Crossref]

10. Anand T, Tanwar S, Kumar R, Meena GS, Ingle GK. Knowledge, attitude and level of physical activity among medical undergraduate students in Delhi. Indian J Med Sci. 2011;4;133-142.

[Crossref]

11. Lobelo F, Duperly J, Frank E. Physical activity habits of doctors and medical students influence their counselling practices. $\mathrm{Br}$ J Sports Med. 2009;43(2)89-92.

doi: $10.1136 /$ bjsm.2008.055426 [Crossref]

12. Padmapriya K, Krishna P, Rasu T. Prevalence and patterns of physical activity among medical students in Bangalore, India. Electron Physician. 2013;5(1)606-610.

doi: $10.14661 / 2013.606-610$ [Crossref]

13. Babakus WS, Thompson JL. Physical activity among South Asian women- a systematic, mixed-methods review. Int J Behav Nutr Phys Act. $2012 ; 9 ; 150$.

doi: $10.1186 / 1479-5868-9-150$ [Crossref]

14. A El-Gilany, Ragaa El-Masry. Physical inactivity among Egyptian and Saudi medical students' Prevent. Med Bullet. 2011;10(1)35-44.

[Crossref] 
15. El-Gilany AH, Badawi K, El-Khawaga G, Awadalla N. Physical activity profile of students in Mansoura University, Egypt. East Mediterr Health J. 2011;17(8)694-702.

[Crossref]

16. Awadalla NJ, Aboelyazed $A E$, Hassanein $M A$, Khalil SN, Aftab R, Gaballa IIet al. Assessment of physical inactivity and perceived barriers to physical activity among health college students, south-western Saudi Arabia. East Mediterr Health J. 2014;20(10)596-604.

[Crossref]

17. Fontes ACD, Vianna RPT. Prevalence and factors related to low level physical activity among university students in a public university in the northeast region of Brazil. Rev Bras Epidemiol. 2009; 12(1)20-29.

doi: $10.1590 / S 1415-790 \times 2009000100003$ [Crossref]

18. Bauman A, Bull F, Chey T, Craig CL, Ainsworth $\mathrm{BE}$, Sallis JF, et al. The International Prevalence Study on Physical Activity- results from 20 countries. Int J Behav Nutr Phys Act. $2009 ; 6 ; 21$.

doi: $10.1186 / 1479-5868-6-21$ [Crossref]
19. Buckworth J, Nigg C. Physical activity, exercise, and sedentary behavior in college students. J Am Coll Health. 2004;53(1)28-34.

doi: $10.3200 / \mathrm{JACH} \cdot 53 \cdot 1.28-34$ [Crossref]

20. Khalaf A, Ekblom Ö, Kowalski J, Berggren V, WestergrenA,Al-Hazzaa $\mathrm{H}$. Female university students' physical activity levels and associated factors- a cross-sectional study in southwestern Saudi Arabia. Int J Environ Res Public Health. 2013;10(8)3502-3517.

doi: $10.3390 /$ ijerph10083502 [Crossref]

21. Bauman A, Allman-Farinelli M, Huxley R, James WPT. Leisure-time physical activity alone may not be a sufficient public health approach to prevent obesity - a focus on China. Obes Rev. 2008;9(1)119-126.

doi: $10.1111 /$ j.1467-789X.2007.00452.x [Crossref]

22. The University of Hong Kong Social Sciences Research Centre. Behavioral risk factor survey (October 2005). Main report, Hong KongGovernment Printing Office. 2005.

Available at accessed 2 July 2014 [Article] [Crossref] 